\title{
Observation of the Hole State Symmetry by Anisotropy of Inelastic Scattering Accompanied by Inner-Shell Excitation
}

\author{
K. Saitoh, ${ }^{*}$ K. Momonoi** and N. Tanaka* \\ * EcoTopia Science Institute, Nagoya University, Nagoya, 464-8603, Japan \\ ** Department of Crystalline Materials Science, Nagoya University, Nagoya, 464-8603, Japan
}

When a fast electron experiences an inelastic scattering accompanied by an inner-shell excitation, the scattering intensity, or differential scattering cross section, shows a momentum-transfer (q) dependence reflecting the symmetry of the initial state $|i\rangle$ and final state $|f\rangle$ of the inner-shell excitation. The anisotropy of $|f\rangle$ can then be studied from the inelastic scattering anisotropy by using an initial state whose symmetry is identified, as some of the examples are shown in Figs 1(a), 1(b) and 1(c). Botton observed the angular distribution of inelastic scattered electrons using a postcolumn energy-filtering system for transmission electron microscopy [1]. This technique allows us a two-dimensional parallel recording of the differential cross section with high q-resolution.

In this paper, we present applications of this technique to materials having anisotropy of their electronic structures. Energy-filtered inelastic scattering patterns were taken by JEOL JEM2010FEF equipped with an omega filter at $120 \mathrm{kV}$, and by FEI Tecnai G2 with Gatan Imaging Filter at $120 \mathrm{kV}$ and $300 \mathrm{kV}$. A series of inelastic scattering pattern were obtained at successive energy losses around corresponding inner-shell excitations with an energy slit with of 1-3 eV.

Figs. 2(a), 2(b), 2(c) and 2(d) show inelastic scattering patterns of $\mathrm{MgB}_{2}$ at energy losses of $180 \mathrm{eV}$ (pre-edge of B $K$-shell excitation), $190 \mathrm{eV}, 198 \mathrm{eV}$ and $204 \mathrm{eV}$ respectively, in which a pre-edge pattern is subtracted as a background. The incident direction was oriented in the [11 $\overline{2} 0]$ direction. The pre-edge pattern (Fig. 2(a)) is isotropic around the central peak, whereas Figs. 2(b) and 2(d) show an anisotropy; the peak is elongated in the $\mathrm{q}_{\mathrm{z}}$ (vertical) direction at $190 \mathrm{eV}$ and in the $\mathrm{q}_{\mathrm{x}}$ (horizontal) direction at $204 \mathrm{eV}$. These anisotropic features are caused by the $|1 s\rangle \rightarrow\left|\pi^{*}\right\rangle$ and $|1 s\rangle \rightarrow\left|\sigma^{*}\right\rangle$ excitations of $\mathrm{B}$, respectively. By assuming that each of the scattering patterns is an incoherent superposition of inelastic scattering patterns accompanied by $|1 s\rangle \rightarrow\left|2 p_{x y}\right\rangle$ and $|1 s\rangle \rightarrow\left|2 p_{z}\right\rangle$ excitations, $\pi$ and $\sigma$ components as a function of energy loss, which can be called as partial EELS spectra [2], were obtained (Fig. 2(e)). A good agreement to the first principle calculation was confirmed [3]. Figs. 3(a), 3(b), 3(c) and 3(d) show inelastic scattering patterns of high- $T_{c}$ superconductor $\mathrm{Bi}_{2} \mathrm{Sr}_{2} \mathrm{CaCu}_{2} \mathrm{O}_{8}$ accompanied by the cupper $L$-shell excitation at energy losses of $925 \mathrm{eV}, 933 \mathrm{eV}, 943 \mathrm{eV}$ and $952 \mathrm{eV}$, respectively. A clear asymmetry due to the excitation of $|2 p\rangle \rightarrow\left|3 d_{x^{2}-y^{2}}\right\rangle$ as a croissant shape can be observed in the pattern at $933 \mathrm{eV}$, which is consistent with previous reports, studied by conventional angular-resolved EELS and XAS [4]. Fig. 3(e) shows partial EELS spectra of the $\mathrm{Cu} 3 d_{x 2-y 2}$ and $3 d_{3 z 2-r 2}$ components determined by the quantitative comparison between the experimental and simulated patterns.

\section{References}

[1] G. A. Botton, J. Electron Spectrosc. Rel. Phenom. 143 (2005) 129.

[2] K. Saitoh, K. Nagasaka and N. Tanaka, J. Electron Microsc. 55 (2006) 281.

[3] R. F. Klie, Y. Zhu, Phys. Rev. B 73 (2006) 014513.

[4] J. Fink et al. J. Electron Spectrosc. Rel. Phenom. 66 (1994) 395. 

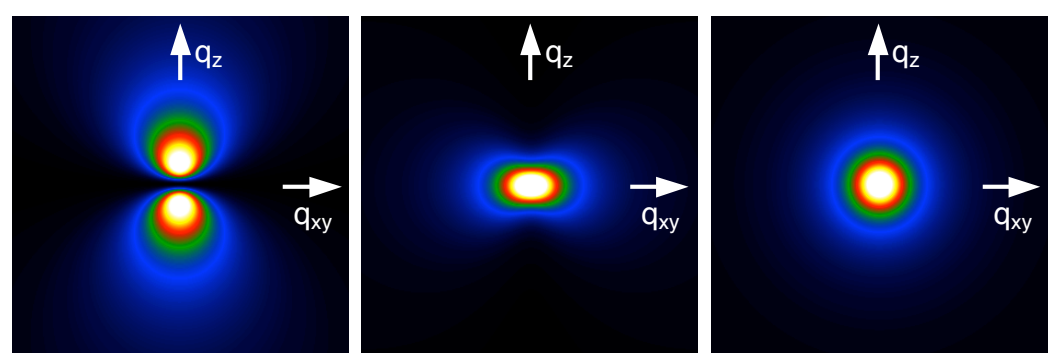

FIG.1. Simulated inelastic scattering patterns of $|1 s\rangle \rightarrow\left|p_{z}\right\rangle$ excitation (a), $|1 s\rangle \rightarrow|\sigma\rangle\left(\left|p_{x}\right\rangle+\left|p_{y}\right\rangle\right)$ excitation (b) and the sum of (a) and (b) (c).
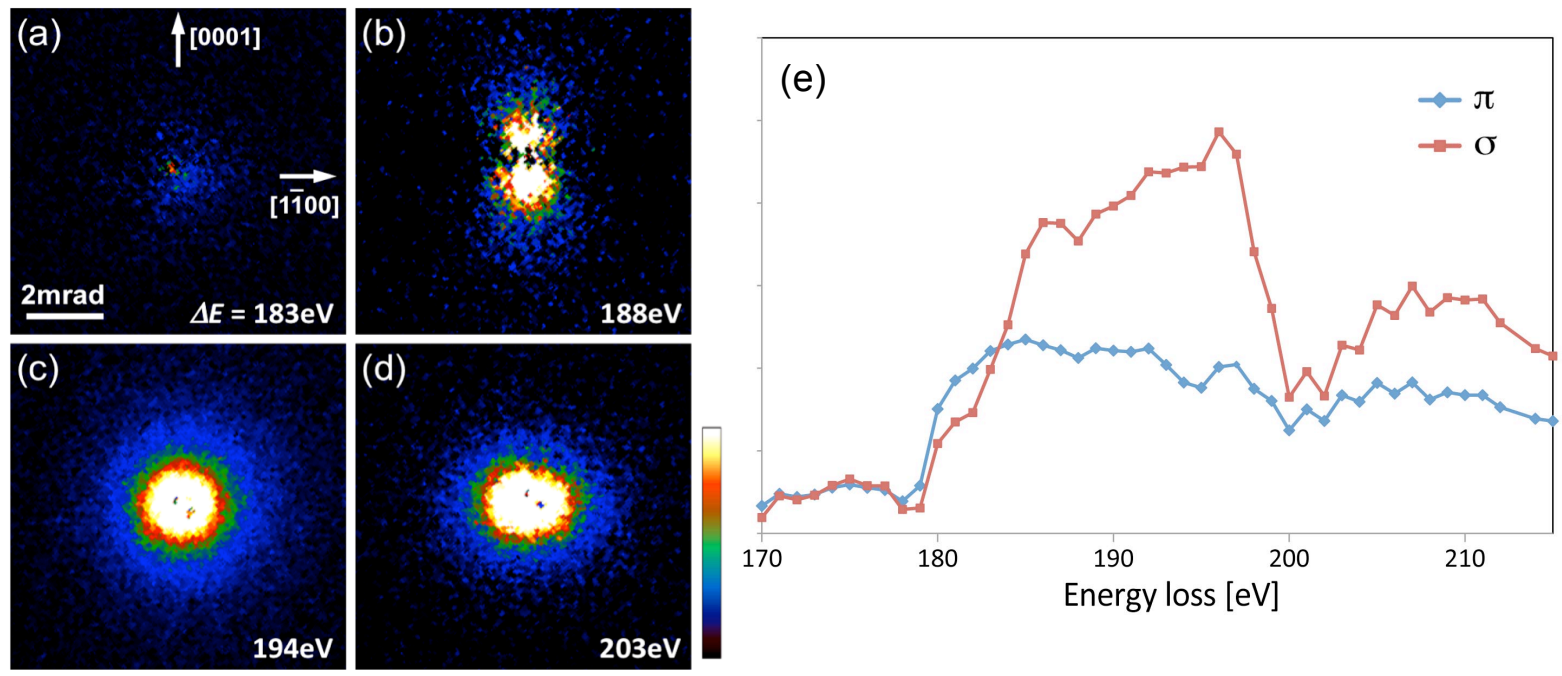

FIG.2. Inelastic scattering patterns of $\mathrm{MgB}_{2}$ accompanied by the $\mathrm{B} K$-shell excitation, in which a pre-edge pattern was subtracted as a background. (a) $\Delta \mathrm{E}=180 \mathrm{eV}$ (pre-edge). (b) $190 \mathrm{eV}$. (c) 198 eV. (d) $204 \mathrm{eV}$. (e) Partial EELS spectra of the B $2 p_{x y}$ (red line) and $2 p_{z}$ (blue line) components.
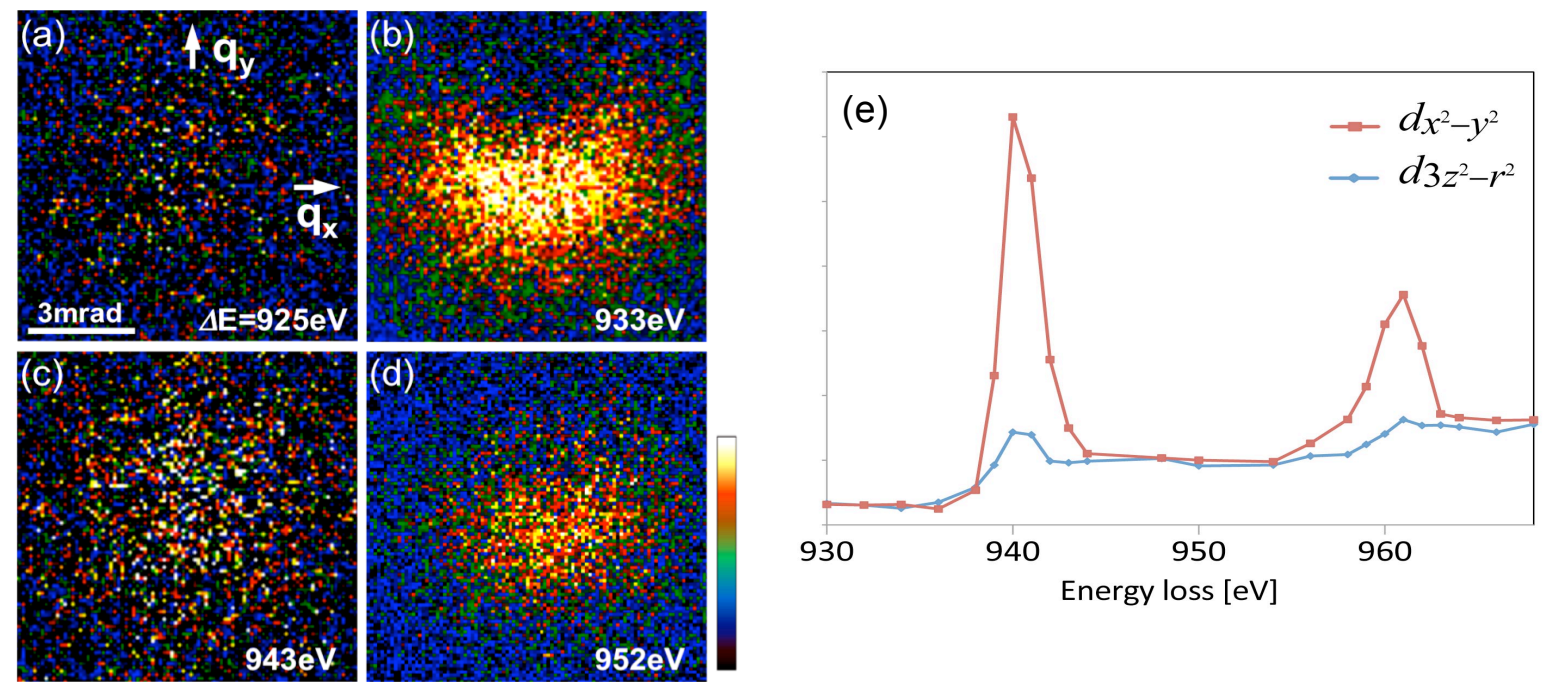

FIG.3. Inelastic scattering patterns of $\mathrm{Bi}_{2} \mathrm{Sr}_{2} \mathrm{CaCu}_{2} \mathrm{O}_{8}$ accompanied by the cupper $L$-shell excitation, (a) $\Delta \mathrm{E}=925 \mathrm{eV}$ (pre-edge). (b) $933 \mathrm{eV}$. (c) $943 \mathrm{eV}$. (d) $952 \mathrm{eV}$. (e) Partial EELS spectra of the cupper $3 d_{x 2-y 2}$ (red line) and $3 d_{3 z 2-r 2}$ (blue line) components. 